\title{
Paediatric immunoglobulin A deficiency revisited
}

\author{
Viktor Gałęcki ${ }^{1 *}$, Michał Janas ${ }^{1 *}$, Piotr Juzyk ${ }^{1 *}$, Radosław Oronowicz ${ }^{1 *}$, Martyna Soloch ${ }^{1 *}$, \\ Wojciech Starczewski ${ }^{*}$, Agata Weigt ${ }^{*}$, Aleksandra Szczawińska-Popłonyk ${ }^{2}$ \\ $12^{\text {nd }}$ year medical student's Research Group, Poznan University of Medical Sciences, Poznan, Poland \\ 2Department of Paediatric Pneumonology, Allergology, and Clinical Immunology, Poznan University of Medical Sciences, \\ Poznan, Poland \\ ${ }^{*}$ These authors contributed equally to this work.
}

\section{ABSTRACT}

Immunoglobulin A (IgA) is the most abundant antibody isotype on mucosal surfaces, with predominantly neutralising non-activating functions, and which plays multifaceted roles in the immune system. IgA protects the host from pathogen invasion in the gastrointestinal and respiratory tracts, enhances diversification of microbiota, and maintains the intestinal homeostasis, exerting its twofold proinflammatory and anti-inflammatory functions by different immune cells and receptors. Therefore, IgA deficiency in children encompasses a broad spectrum of clinical phenotypes including infectious, allergic, autoimmune, and inflammatory conditions. In this review we also provide new insight into the genetic background and immunopathology of IgA deficiency in children.

KEY WORDS:

IgA deficiency, children, T cells, B cells, immunity.

\section{INTRODUCTION}

Immunoglobulin A $(\operatorname{Ig} \mathrm{A})$ is the most abundant antibody isotype present on mucosal surfaces in the human body. IgA deficiency (IgAD) can be categorised as absolute (total), partial, or selective (SIgAD). Absolute IgAD is defined as an undetectable serum IgA level, below $0.005 \mathrm{~g} / \mathrm{l}$, whereas partial IgAD signifies an IgA level that is two standard deviations below age-adjusted mean. Selective IgA deficiency (SIgAD) is the most prevalent primary immunodeficiency in Caucasian populations, defined by serum level of $\operatorname{IgA}<0.007 \mathrm{~g} / \mathrm{l}$, with a prevalence of approximately $1: 600$ in the population $[1,2]$. In children, according to the current ESID recommendation, a definitive diagnosis of absolute IgA deficiency is estab- lished beyond the age of four years because of delayed maturation of this immunoglobulin isotype $[1,2]$ and concomitantly, in young children below four years of age, the IgA serum level lower than $0.007 \mathrm{~g} / \mathrm{l}$ has no practical and prognostic significance.

IgA plays a vital role in defending mucous membranes especially in the airways and in the intestines, where production of IgA reaches up to $5 \mathrm{~g}$ a day [3]. Mucosal secretion contains almost only IgA, while IgG is the most abundant in blood. IgA in mucosal secretion is referred to as secretory $\operatorname{IgA}(\mathrm{SIgA})$, which performs a plethora of functions, such as immune exclusion of pathogens and toxins, neutralisation of intracellular pathogens, downregulation of pro-inflammatory responses, blocking symbiotic bacteria from passing through an epithelial layer, and oversee-

\section{ADDRESS FOR CORRESPONDENCE:}

Aleksandra Szczawińska-Popłonyk, Department of Paediatric Pneumonology, Allergology, and Clinical Immunology, 27/33 Szpitalna St., 60-572 Poznan, Poland, ORCID: 0000-0001-7244-1882,

e-mail: ola@malwa.com.pl 
ing of homeostasis of commensals [4]. The second type is serum IgA, the function of which is still mostly unknown although it has been suggested that it might work against pathogens and, in lower concentrations, downregulate the proinflammatory responses due to regulation of cytokines and effector cells function [5]. The difference between secretory and serum types of IgA is that the latter is monomeric and SIgA is dimeric, connected by the J chain, and contains a secretory component (SC), an extracellular portion of polymeric immunoglobulin receptor ( $\mathrm{pIgR}$ ) that transfers IgA across epithelial cells [6].

In the intestine, the most efficient stimulations of immune responses are generated in gut-associated lymphoid tissue (GALT), a site for B cell activation and proliferation in response to antigens. The most important for the IgA part of GALT is its inductive site, where most IgAs are generated in Peyer's patches (PP), already present at birth and in isolated lymphoid follicles (ILF), which are developed in infants after gut colonisation by bacteria. One of the most important immune responses is the significant production of SIgA by plasma cells residing in the lamina propria (LP) of the intestinal mucosa [7]. The synthesis of IgA in intestines is not entirely known; notwithstanding, it is believed to follow two pathways: T-cell dependent and T-cell independent. The T-cell-dependent pathway is due to the generation of T-follicular helper cells (Tfh) and the appearance of germinal centres (GC) in the PPs. Follicular $\mathrm{T}$ helper cells in the gut arise preferentially from regulatory $\mathrm{T}$ cells with the expression of a transcription factor forkhead box protein 3 (Foxp3), and they also show an expression of the chemokine receptor 5 (CXCR5). In GC they are localised in the proximity of B cells and assist immunoglobulin class switching from IgM+B cells expressing activation-induced cytidine deaminase (AID) to IgA-producing B cells [8]. The T-cell-independent switching from $\operatorname{IgM}$ to $\operatorname{IgA}$ antibodies synthesis in B cells is sited in the ILFs [9] and depends mostly on the subset of specialised epithelial microfold (M) cells, which bears a fractalkine CX3CR1 receptor and is equivalent to monocyte-derived dendritic cells. These cells show the capability to uptake pathogens and activate B cells due to secretion of interleukin (IL)-10, IL-6, tumour necrosis factor $\alpha$ (TNF- $\alpha$ ), B-cell activating factor (BAFF), and a proliferation-inducing ligand (APRIL) resulting in IgA synthesis in AID expressing B cells [7]. In the pathogenesis of $\operatorname{IgAD}$, heterogeneous mechanisms are suggested, affecting both intrinsic terminal maturational B-cell defects and abnormal immunoglobulin A secretion, impaired T-cell-dependent support of class switching, memory B cell, and plasma cell development, as well as the impairment of cytokine activity.

\section{GENETICS OF IGA DEFICIENCY}

The mutations in human leukocyte antigen (HLA) locus have been shown to be associated with IgAD. The extended HLA-A*01-B ${ }^{\star} 08-\mathrm{DRB} 1^{\star} 0301-\mathrm{DQB} 1^{\star} 02(\mathrm{DR} 3)$ haplotype was found to be the strongest genetic risk factor for IgAD in Northern European populations, in which $13 \%$ of DR3 homozygotes developed IgAD. On the other hand, the HLA-DRB1*1501-DQB1 ${ }^{\star} 06$ (DR2) haplotype displayed a strong protective effect against developing $\mathrm{IgAD}$, with homozygotes showing almost complete resistance to the disease. HLA-B ${ }^{\star} 14-\mathrm{DRB} 1^{\star} 0102-\mathrm{DQB} 1^{\star} 05$ (DR1) and the HLA-B ${ }^{\star} 44-\mathrm{DRB1}{ }^{\star} 0701-\mathrm{DQB}^{\star} 02$ (DR7) haplotypes also presented an increased risk of developing IgA deficiency. Butyrophilin-like 2 (BTNL2)-HLA-DRA loci on the telomeric end of class II of the major histocompatibility complex (MHC) region and in the HLA-DQB1 region were also considered to be risk factors, further confirming the susceptibility of IgAD to multilocal effects, spanning the entirety of the HLA region $[10,11]$. There are also associations of polymorphisms in non-HLA loci, causing an increased risk of IgAD. Interferon induced with helicase C domain 1 (IFIH1) gene, mutations of which have previously been associated with type 1 diabetes and systemic lupus erythematosus, has a rare variant (p.Ile923 Val) showing an increased risk of IgAD development.

Not only mutations in IFIH1 loci but also in Pvt1 oncogene (PVT1), autophagy-related 13-autophagy, and Beclin 1 regulator 1 (ATG13-AMBRA1) and C-type lectin domain containing 16A (CELC16A) have been proven to be associated with a higher risk of IgAD [12]. The transmembrane activator and calcium modulator ligand (CAML) interactor (TACI), encoded by the TNF receptor superfamily member 13B (TNFRSF13B)/TACI gene mutation was found in a group of patients with common variable immunodeficiency (CVID) and IgAD. The percentage of mutation in this gene for SIgAD was $2.7 \%$ and for partial IgAD was $2.9 \%$, and the control group of healthy individuals showed no mutation in TNFRSF13B [13]. Multiple genetic mutations as a cause of IgAD suggest that it may influence entire families, as was shown in the study by Karaca et al., who revealed that in first-degree relatives of IgAD patients up to $33.6 \%$ were affected by IgA production defects, whereas $19.1 \%$ had IgA levels lower than the age-adjusted mean. The risk of carrying IgAD in relatives of affected patients is estimated to be up to $20 \%$ [14].

\section{IGA IN SYSTEMIC AND MUCOSAL IMMUNITY}

$\operatorname{IgA}$ is the most abundant antibody class in the human immune system, concentrated mostly at mucosal surfaces. The main function of IgA is to preserve the homeostatic condition of gut microbiota. The importance of SIgA is shown in the very high amount of SIgA in the colostrum, thus performing an important role in newborn immunity of gastrointestinal and respiratory tracts [15]. Produced in the mucosal immune system such as GALT by plasma cells in organised GCs such as PPs and ILFs in the intestinal LP, polymeric IgA containing a $J$ chain is bound to a polymeric IgA receptor ( $\mathrm{pIgR}$ ) present in the basolateral 
membrane of epithelial cells. The complex ligand-pIgR is transported to the apical plasma membrane and released to the intestinal lumen after cleavage by an endoprotease, thus producing SIgA from pIgA-pIgR complex and the free secretory component (SC) from spare pIgR [16]. The free SC provides stability to SIgA by a covalent bond. Monomeric IgA is present mostly in serum in lower concentrations and plays a significant role in immunity against pathogens. A well-known function of IgA is to provide passive immunity at the mucosal surfaces. In the lumen of intestines, SIgA molecules can directly block the access of pathogens such as bacteria, viruses, protozoa, and toxins to host cells via antigen binding sites during the processes of "immune exclusion" [4]. This is made up of agglutination or clumping of pathogens as a result of antibody binding to corresponding antigens, entrapment in mucus, and clearance of pathogens and antigens through peristalsis, thus creating the first line of defence in GALT.

Both polymeric SIgA, its secretory component, and the residual polypeptide from the pIgR are highly glycosylated, and these carbohydrate chains have been shown to play an antigen epitope-independent, glycan-dependent, noncanonical role in intestinal immune homeostasis. Moreover, the glycosylated chains of SIgA may prevent SIgA proteolysis by microbiota proteases in the lumen of intestines. They have been shown to interact with pathogenic bacteria by competition of adhesion or by fixing SIgA to the mucus layer and having a functional protective effect. These SIgA glycosylated chains also alter bacterial metabolism and gene expression and can decrease bacterial motility by interfering with flagella [17]. In neonates, a limited exposure of tissues to taxes of intestinal microbiota is achieved due to the protective effect of IgA antibodies present in maternal milk, which delay the infant's own IgA production, but they also beneficially shape the composition of its intestinal microbiome [17]. IgA might have a direct effect on bacterial virulence. The study by Mantis et al. [18] on an animal model showed that the binding of a murine monoclonal IgA (IgAC5) to the O-Ag of Shigella flexneri suppressed the activity of the bacterial type 3 secretion system (T3S), which is required for them to gain entry into the intestinal epithelial cells for bacteria, thus reducing the virulence of the bacteria due to intracellular neutralisation, elimination, and excretion of immune complexes. Molecules of dimeric IgA during pIgR-related transcytosis are able to neutralise endocytosed bacterial lipopolysaccharide and also can block viral infections in epithelial cells, facilitating pIgR in transporting the IgA-antigen complex across epithelial cells into the lumen of the intestine. Mantis et al. in the above-mentioned study [18] showed new immunopotentiating properties of SIgA in mucus, in the process of the retro-transport of SIgA-based immune complexes by $\mathrm{M}$ cells of PPs to the subepithelial dome of the intestinal mucus, which may trigger specific mucosal and systemic immune responses. The authors demonstrated that oral delivery of recombinant SIgA (rSIgA) composed of a mouse pIgA and a human SC, triggered a T-cell proliferation in the mesenteric lymph nodes and in the spleen. Moreover, rSIgA immunisation induced Th1/Th2-based tolerance to immune responses. Furthermore, they observed rSIgA-induced migration of dendritic cells (DCs) from the subepithelial dome to the interfollicular dome, pointing to the activation of DCs. In addition to the glycan-dependent IgA immune functions and its binding to the pIgR, as discussed above, a crucial point in this regard is its canonical interaction with the FcaRI receptor (or CD89), which is presented on neutrophils, eosinophils, monocytes, and macrophages, such as Kupffer cells in the liver or alveolar macrophages in the lungs, platelets, and DCs. This FcaRI receptor itself lacks signalling motifs in its cytoplasmic part and can thus transmit signals by association with the $\mathrm{Fc} \gamma \mathrm{R}$ chain [19]. It means that IgA-dependent cytokine production is not caused by one individual receptor but by cooperation with other receptors, primarily pattern-recognition receptors (PRR), to increase or decrease the production of specific cytokines [19]. Interactions of polymeric IgA with FcaRI trigger the pro-inflammatory properties. During infection molecules of IgA aggregate, creating complexes, and bind FcaRI with higher affinity than monomeric or dimeric IgA, thereby inducing classic ITAM (immune tyrosine-based activation motifs) signalling and amplifying inflammation through interaction with PRRs [20]. This binding leads to a wide spectrum of cellular responses depending mostly on cellular diversity, including: antibody-dependent cell-mediated cytotoxicity (ADCC), release of cytokines, degranulation, respiratory burst and superoxide generations, and phagocytosis. Although FcaRI amplify several inflammatory processes, it also has anti-inflammatory properties. Immunosuppressive ITAMi (immune tyrosine-based activation motifs inhibitor) is triggered by monomeric and dimeric interaction with IgA but with an average affinity. It is worth noting that these phenomena function in homeostatic conditions, when IgA complexes are not observed, and therefore this creates the possibility of coexistence between microbiotas and the host by preventing FcaRI-induced cytokine production. On the other hand, an anti-inflammatory feature of the IgA antibody is its insufficient interaction with the complement. $\operatorname{IgA}$ deficit of the C1q binding motif prevents activation of the complement via the classic pathway. Although polymeric IgA is able to bind the mannose-binding lectin (MBL) and to activate the complement via the lectin pathway, in some situations where the antigen is limited, IgA can even limit the $\mathrm{C} 1 \mathrm{q}$ component by preventing the binding of more powerful complement activators, such as IgM or IgG. Hence, in IgA deficiency due to an impaired multidirectional immunosurveillance, diverse disorders of the local and systemic immune homeostasis, with excessive or insufficient proinflammatory and anti-inflammatory responses, may be expected [20]. 


\section{THE B-CELL AND T-CELL DEFECTS IN IGA DEFICIENCY}

While there are numerous reports considering the abnormalities in $\mathrm{B}$ and $\mathrm{T}$ lymphocyte subpopulations in common variable immunodeficiency (CVID) [21, 22], it is still relatively unclear what changes occur in the composition of T cells and B cells in SIgAD. Because these two disorders are assumed to have a common genetic basis [23] and the progression of SIgAD into CVID was described, alterations in $\mathrm{B}$ cells and $\mathrm{T}$ cells are expected to be similar in these two conditions. Therefore, it could be assumed that impairments of immunological mechanisms in CVID, like defective B cell class switching and differentiation [24] or activation of $\mathrm{T}$ cells [25], are also present in SIgAD, or even similar genetic mutations occur [26]. Some of these assumptions were proven in SIgAD although to a lesser extent than in CVID. As stated above, $B$ cell class switching and differentiation might be impaired in SIgAD because it occurs in CVID. Indeed, it was observed that CD27+ IgD- IgM+-switched memory $B$ cells had a tendency to decrease in IgAD [22, 27], although not as drastically as in CVID. However, in patients affected with SIgAD, the number of memory B cells might be clinically more important than the level of IgA in the serum itself.

While Litzman et al. [22] reported that B-cell differentiation in SIgAD patients shows no abnormalities, Lemarquis et al. [27] suggested that a defective B cell response to TLR9 leads to a reduction in differentiation to $\mathrm{CD} 27+\mathrm{IgD}$ - switched memory B cells, and also their precursors - CD24hiCD38hi transitional B cells. Because TLR9 is a strong inductor of IL-10 production by transitional B cells, which in turn stimulates IgA production, the authors hypothesise that a defective $B$ cell response to the receptor might cause decreased IgA levels in $\mathrm{S}$ IgAD patients. Furthermore, studies report that absolute and relative numbers of B lymphocytes in SIgAD were comparable to healthy controls [22], whereas surface expression of IgA on B cells were significantly lower in SIgAD [27]. In comparison, CVID patients had significantly lower numbers of $\mathrm{CD} 27+\operatorname{IgD}-\operatorname{IgM}+$ switched memory $\mathrm{B}$ cells and B-cell differentiation was defective at every stage $[22,24]$.

Alongside B-cell defects, CVID also shows a tendency to alter the T-cell compartment and the mutual relationship between T-cell subsets as well T-cell functions. The $\mathrm{T}$ cells of CVID patients may present spontaneous and accelerated apoptosis or have reduced mitogen-induced proliferation [28]. Moreover, many studies agree that there are lower $\mathrm{CD} 4+$ and $\mathrm{CD} 8+$-naïve $\mathrm{T}$ cell absolute numbers and proportions in CVID [22, 25, 29] and lower numbers of T regulatory cells (Treg) [25, 29]. In contrast, increased activation of $\mathrm{T}$ cells was reported (CD4+CD25+ or CD95- and HLA-DR+-expressing T cells) [22], whereas relative numbers of cytotoxic $\mathrm{CD} 8+\mathrm{T}$ cells increased and absolute numbers of CD4+ T helper cells decreased in CVID patients. In the case of SIgAD, some studies reported that the $\mathrm{T}$ cells seem to follow several of these patterns, to a lesser extent however than in CVID [22, 25], while others did not show any significant changes in the T cell compartment in SIgAD [27, 29]. Nechvatalova et al. [25] found that the naïve CD4+ T cell percentage was decreased in SIgAD patients, even if this deficiency was not as profound as in CVID patients. These authors further reported a significant increase in terminally differentiated T cells in both SIgAD and CVID patients, especially of the $\mathrm{CD} 8+$ subset in CMV-seropositive compared to CMVseropositive healthy controls. Interestingly, SIgAD patients in this study also manifested an increased percentage of Treg cells in comparison to healthy controls. Likewise, Litzman et al. [22] reported a significant increase in the relative number of $\mathrm{CD} 8+\mathrm{T}$ cells and a significant decrease in the absolute number of CD4+ $\mathrm{T}$ cells in SIgAD patients in comparison to healthy controls, while having a decreased relative amount of CD8+ T cells and an increased relative amount of $\mathrm{CD} 4+\mathrm{T}$ cells in comparison to CVID patients. Surprisingly, in this study, the authors reported a decrease in CD95-HLA-DR+-activated T cells, and an increase in CD4+CD25+-activated $\mathrm{T}$ cells in comparison to both healthy controls and CVID patients. Furthermore, they observed no other abnormalities in the T-cell activation markers in SIgAD. Understanding changes in the function and relative proportions of B-cell and T-cell subsets in SIgAD may prove to be of important clinical value. Because the progression of SIgAD to CVID has been recorded [23] and both conditions seem to have similar T-cell and B-cell dysfunctions, the intensity of the changes could be a useful diagnostic tool in tracking progression. Furthermore, as was reported by Warnatz et al. [24], the proportion of switched memory B cells in $\mathrm{PB}$ cells could be connected to the severity of clinical manifestations and complications in SIgAD.

In addition, other primary immunodeficiency diseases (PIDs) such as ataxia telangiectasia or DiGeorge syndrome (22q11.2 deletion syndrome), in which IgA deficiency is a part of the immunophenotype, were also reported to alter the lymphocyte population. Patients suffering from ataxia telangiectasia, similarly to SIgAD patients, had a decreased proportion of both naïve $B$ and T cells and switched memory B cells [30]. T-cell lymphopaenia and a diminished proportion of switched memory B cells were also found in DiGeorge syndrome patients [31]. These findings suggest that similar lymphocyte impairments are involved in various PIDs, and such awareness may prove critical in assessing these conditions.

\section{DISRUPTED INTESTINAL HOMEOSTASIS IN IGA DEFICIENCY}

IgA is a dominant immunoglobulin class secreted in the intestinal mucosa and plays an important role in 
maintaining eubiosis in the gut. While germ-free animals present almost no IgA production, its synthesis is induced rapidly after bacterial colonisation of the intestine [32]. IgA arises from B cells, which undergo somatic hypermutation (SHM), responsible for affinity maturation, and class switch recombination (CSR), which alters the effector function of immunoglobulins [33]. Despite its function of neutralising pathogens and toxins, IgA plays a key role in shaping the composition of beneficial gut microbiota. In the activation-induced cytidine deaminase (AID) deficiency both SHM and CSR are disrupted, which leads to the expansion of anaerobic microbes in the small intestine, ILF hyperplasia, and GC enlargement. It has also been observed that IgM is secreted in $\operatorname{IgAD}$, but it does not prevent the expansion of anaerobes. Likewise, the AIDG23S single point-mutation, which inhibits SHM, while CSR is preserved, also leads to intestinal dysbiosis and the disruption of microbiome homeostasis [34]. Interestingly, IgA might be used by some commensal bacteria to maintain colonisation. Bacteroides fragilis has a genetic locus called the commensal colonisation factor, which regulates expression of polysaccharides that contribute to IgA binding. This IgA coating promotes extensive colonisation and single-strain stability. It has been shown that the IgA antibody shapes the microbiome composition, promoting gut colonisation by some taxa, while protecting the intestinal barrier from others [35]. The composition of gut microbiota has also been studied in SIgAD patients, showing that only discrete bacterial populations are affected, whereas global diversity is preserved. However, anti-inflammatory species of bacteria, which are IgA targeted in healthy controls, are more likely to be depleted and proinflammatory species are overrepresented in SIgAD patients. The authors of the study also point out an ectopic localisation of oral microbiota in the lower digestive tract, which may also trigger an inflammatory reaction. The study also confirmed that in SIgAD some bacteria are bound by $\operatorname{IgM}$, but that pattern of binding does not fully overlap with that of IgA, and this contributes to disturbing the bacterial dependency association network in SIgAD [36]. In this context an important issue is the link between IgA deficiency and inflammatory bowel disease (IBD). IgA-deficient patients are reported to have a higher risk of developing Crohn's disease and ulcerative colitis. In an experiment in which gnotobiotic mice (an animal with known flora) were infected with bacteria from IBD patients, it was proven that the bacteria that were IgA coated were especially able to cause colitis. However, these bacteria were not able to cause colitis on their own, and a specific environmental factor was needed for the disease to develop. The authors suggest that even though the host produces IgA to neutralise these proinflammatory colitogenic strains, the reaction is not sufficient, which leads to the development of IBD, and whether in IgAD the inflammatory response to IgA inducing bacteria is even higher still needs to be investigated [37].

It has also been reported that celiac disease (CD) has a higher prevalence in IgA-deficient children than in the general population. This phenomenon is explained by the common genetic background of both diseases, which is associated with the HLA-haplotype. Such a concomitance is the reason why, along with IgA antibodies against tissue transglutaminase (tTG), the total IgA level should also be measured when diagnosing $\mathrm{CD}$, in order to avoid false negative results. Therefore, in IgA-deficient children antigen-specific anti-endomysium (EMA), anti-tTG, or anti-deamidated gliadin peptides (DGP) IgG antibodies should be tested [38]. However, in partial IgA deficiency anti-tTG IgA seems to be sensitive enough to be used for ruling out $\mathrm{CD}$. Patients with $\mathrm{CD}$ and SIgAD tend to acquire other autoimmune diseases more often and lack the classic gastrointestinal symptoms of CD [39].

\section{CLINICAL PHENOTYPES IN IGA DEFICIENCY}

IgAD encompasses miscellaneous phenotypes. Even though most of the cases (approximately two-thirds) are asymptomatic, some affected individuals are characterised by susceptibility to more frequent respiratory or gastrointestinal tract infections (RT and GIT, respectively) and various allergic reactions [40]. The differentiating factors in the manifestations of this disorder are principally the age of the patients, because paediatric and adult patients can present in different ways, as well as concomitant immunological impairments [41]. The type of deficiency - partial or absolute - is often a factor determining the clinical course of IgAD, but in some patients it seems to be of little importance [2].

In symptomatic paediatric patients IgAD most often (in nearly $95 \%$ of IgA deficient individuals) manifests clinically as infections of the upper or lower respiratory tract [41]. From $40 \%$ to $90 \%$ of the cases are diagnosed only because of infectious sinopulmonary disorders, and these sometimes remain the only remarkable symptoms. The frequency of infections is greater with simultaneous abnormalities of IgG subclasses, particularly with coexisting IgG2 and IgG3 deficiencies [42]. The infections are frequently prolonged and recurrent with numerous contributing bacteria, especially Streptococcus pneumoniae, Haemophilus influenzae, and Staphylococcus, but also atypical and opportunistic ones such as Mycoplasma or Pseudomonas aeruginosa [41]. Infections may result from an impaired immune response to vaccination noted in IgA-deficient children and adults who have been administered an unconjugated polyvalent pneumococcal vaccine [43]. IgA deficiency lower than $0.007 \mathrm{~g} / \mathrm{l}$ is an established risk factor for the incidence of pneumonia and may predispose affected individuals to pneumococcal infections of the RT, and it also implies rhinosinusitis and tonsillitis [44]. Eventually repeated infections of the 
airways due to continuous inflammation and the presence of bacterial agents can initiate a remodelling of the bronchial mucosa as well as pulmonary damage and evolve into chronic respiratory disorders, which reflects a pathophysiological vicious circle of infection and inflammation [40, 41]. Some IgAD children develop bronchiectasis, an irreversible dilation of the airways, caused by the inflammatory destruction of the bronchial walls; however, this is less common than in other immunodeficiencies, such as CVID or X-linked agammaglobulinaemia (XLA) [41]. Furthermore, an investigation of IgA- and IgM-deficient individuals revealed that the coexistence of these two disorders showed a statistically significant increase in the prevalence of bronchiectasis in comparison to normal antibody levels or to each of these deficiencies alone [45]. Likewise, IgA deficiency and concomitant IgG subclass abnormalities can induce the development of interstitial lung disease (ILD), with ground-glass opacities, pulmonary nodules, and mediastinal lymphadenopathy [40, 46]. Moreover, the coexistence of pulmonary hemosiderosis in children along with IgAD has been reported [47], and thus apparently the impairment of IgA production can underlie this condition.

Because IgA is the most abundant immunoglobulin isotype on the surface of tracheobronchial and gastrointestinal mucosa, IgAD enhances the penetration of allergens through RT and GI mucous membranes. In the studies by Erkoçoğlu et al. [48] and Özcan et al. [49] IgA deficiencies left the bronchial mucosal lining defenceless to allergens, predisposing children to bronchial hyperreactivity. There is also a significant relationship between SIgAD and ensuing allergic symptoms, especially asthma, in children, in whom these conditions coincided in 35\% of cases [48]. Furthermore, Özcan et al. established that there is no notable difference in the frequency of occurrence of asthma between the groups of paediatric patients with selective or partial IgA deficiency [50]. Nevertheless, reports of wheezing are significantly remarkable in IgA-deficient patients compared to other primary antibody production defects, especially IgG subclass deficiency [50]. In comparison to the latter antibody deficiency, bronchial hyperreactivity is also notably more frequently assessed in both partial and selective IgA production defects [49]. Notwithstanding, unlike the aforementioned reports, in the study by Urm et al. [51] it was shown that a prior diagnosis of asthma tends to increase a contingency of recognition of SIgAD or CVID. This association between asthma and antibody production defects presumably helps to explain the predisposition of asthmatic patients to recurrent bacterial infections of the airways and infection-triggered exacerbations [51]. Apart from asthma, many IgA-deficient patients develop allergic rhinitis, atopic dermatitis, or conjunctivitis and show vulnerability to food allergens [52]. Altogether, approximately four out of 10 of these individuals present allergic manifestations [48]. Except for food allergy-related gastroin- testinal manifestations, such as abdominal colic, chronic diarrhoea, poor weight gain, and atopic dermatitis, other symptoms of SIgAD comprise gastrointestinal infections [53]. The defective production of mucosal IgA2 isotype of this antibody, as well as the frequent use of antibiotics due to common respiratory infections, can lead to alterations of gut microbiome and decrease antipathogenic resistance [40]. In paediatric patients with low serum and secretory IgA levels, prolonged infections of the intestines caused by Adenovirus or Norovirus [53] as well as Giardia intestinalis occur, which, along with simultaneous uncontrolled inflammation, poses a risk of potential exacerbation and the development of chronic enteropathy.

\section{AUTOIMMUNITY AND MALIGNANCY IN IGA DEFICIENCY}

There is a likely correlation of SIgAD and autoimmune diseases, which has been the subject of multiple studies over the last few years. The link might be due to the shared genetic background of IgAD and autoimmune diseases. SIgAD is related to the genes within the MHC region, and a strong relationship has been shown between the very conservative ancestral haplotype 8.1 (HLA-A1, B8, Dr3, DQ2) and several autoimmune disorders, such as Graves' disease (GD), systemic lupus erythematosus (SLE), type 1 diabetes (T1D), and CD [54]. Autoimmune diseases and SIgAD also share a genetic background, which involves non-MHC encoding genes such as IFIH1 and CLEC16A, and there is the possibility of an epigenetic and post-infectious immunopathogenetic effect. In SIgAD lacking secretory IgA2 subclass, the processing and clearing of external antigens at mucosal surfaces is defective, which may lead to the deposition of immune complexes, inflammatory tissue damage, and potentially the breakdown of immune tolerance, resulting in the formation of self-reactive clones of lymphocytes, cytokine dysregulation, and the generation of anti-tissue antibodies [54].

Autoimmunity is a clinically important issue in both paediatric and adult SIgAD, and its prevalence varies from $7 \%$ up to $36 \%$ of affected individuals [55]. While a strong association between SIgAD and systemic autoimmune diseases is observable, such as SLE and juvenile idiopathic arthritis (JIA), RA, psoriasis, and sarcoidosis $[54,55]$, the co-occurrence of miscellaneous organ-specific autoimmune disorders with SIgAD has become more evident. These comprise haematological manifestations, most frequently autoimmune haemolytic anaemia, endocrine disturbances, such as T1D and autoimmune thyroiditis, alopecia, and autoimmune enteropathies. In a clinical study by Sparchez et al. [56], which was performed on a cohort of paediatric PID patients, the associated autoimmune conditions in children affected with SIgAD included mixed connective tissue disease, autoimmune hepatitis, psychotic episodes, aseptic necrosis of the hip, dermatomyositis, and autoimmune hepatitis. 
It must be stressed that in children affected with SIgAD the onset of juvenile autoimmune phenomena may be variable, and they may develop as early as in infancy, and the clinical course may be severe, aggressive, and complicated by overlapping syndromes $[56,57]$. On the other hand, it must be taken into consideration that IgAD can also arise in adolescents and does not necessarily have a genetic background, but may also be secondary as a result of medication used in the treatment of autoimmune diseases, among them RA, with drugs such as D-penicillamine, corticosteroids, sulfasalazine, and gold salts [58].

While IgA plays a crucial role in the maturation and regulation of the intestinal microbiota, it may be assumed that SIgAD leads to perturbances in homeostasis of the intestinal microbiome, increased growth of colopathogenic bacterial strains and enhanced susceptibility to intestinal damage, which further leads to inflammation and to autoimmune inflammatory bowel diseases, such as Crohn's disease or ulcerative colitis, and early-onset enteropathies in the paediatric SIgAD population [59]. It has also been shown that IgAD slightly increases the risk of gastrointestinal malignancy, and that this observation might be related to the destruction of mucous membranes by various pathogens leading to infections and resulting in chronic inflammatory lesions of the digestive tract. In summary, it must be stressed that non-classical phenotypes with autoimmune, chronic inflammatory, and lymphoproliferative disorders are important warning signs of the primary IgA production defect. It is worth noting that in the search for a common immunopathogenesis of autoimmune and inflammatory disorders associated with SIgAD in children, abnormalities of Foxp3 expressing Treg cells were noted [60].

\section{CONCLUSIONS}

IgA plays a multifaceted role in the human immune system, being crucial for the mucosal and systemic defence against pathogens, interacting with intestinal microbiota and enhancing its diversity, thus maintaining homeostasis in the gut, as well as exerting bidirectional anti- and proinflammatory effects on the immune cells. The IgA antibody protective functions are hence not confined to inhibiting infections, but also rely on suppressing the development of autoimmune, allergic, and inflammatory conditions. While in children the most extensive immune expansion, maturational shifts within lymph cell subsets and acquisition of immune competence, occur during the first five years of life, young children with mild transient IgA hypogammaglobulinaemia may share a similar immunophenotype with these paediatric patients, in whom an absolute SIgAD with early onset of systemic complications develops. Furthermore, asymptomatic IgA-deficient children show the susceptibility to infections and organ-specific immunopathology and thus require regular monitoring. This issue creates important diagnostic and clinical dilemmas in the population of paediatric patients with IgA production defects. The constellation of clinical manifestations in SIgAD children implies the necessity for individualised multispecialist care with the active participation of, but not limited to, pulmonologists, laryngologists, allergologists, gastroenterologists, rheumatologists, endocrinologists, and stomatologists, under the supervision of a clinical immunologist.

\section{DISCLOSURE}

The authors declare no conflict of interest.

\section{REFERENCES}

1. Moschese V, Chini L, Graziani S, et al. Follow-up and outcome of symptomatic partial or absolute IgA deficiency in children. Eur J Pediatr 2019; 178: 51-60.

2. Seidel MG, Kindle G, Gathmann B, et al. ESID Registry Working Party and collaborators. The European Society for Immunodeficiencies (ESID) Registry Working Definitions for the Clinical Diagnosis of Inborn Errors of Immunity. J Allergy Clin Immunol Pract 2019; 7: 1763-1770.

3. Fagarasan S, Honjo T. Intestinal IgA synthesis: regulation of frontline body defenses. Nat Rev Immunol 2003; 3: 63-72.

4. Corthésy B. Multi-Faceted Functions of Secretory IgA at Mucosal Surfaces. Front Immunol 2013; 4: 185.

5. Leong KW, Ding JL. The Unexplored Roles of Human Serum IgA. DNA Cell Biol 2014; 33: 823-829.

6. Johansen FE, Braathen R, Brandtzaeg P. Role of J chain in secretory immunoglobulin formation. Scand J Immunol 2000; 52: 240-248.

7. Suzuki K, Kawamoto S, Maruya M, et al. GALT: organization and dynamics leading to IgA synthesis. Adv Immunol 2010; 107: 153-185.

8. Tsuji M, Suzuki K, Kitamura H, et al. Requirement for lymphoid tissue-inducer cells in isolated follicle formation and $\mathrm{T}$ cell-independent immunoglobulin A generation in the gut. Immunity 2008; 29: 261-271.

9. Tsuji M, Komatsu N, Kawamoto S, et al. Preferential generation of follicular B helper T cells from Foxp3+ T cells in gut Peyer's patches. Science 2009; 323: 1488-1492.

10. Ferreira RC, Pan-Hammarström Q, Graham RR, et al. High-density SNP mapping of the HLA region identifies multiple independent susceptibility loci associated with selective IgA deficiency. PLoS Genetics 2012; 8: e1002476.

11. Kralovicova J, Hammarström L, Plebani A, et al. Fine-scale mapping at IGAD1 and genome-wide genetic linkage analysis implicate HLA-DQ/DR as a major susceptibility locus in selective IgA deficiency and common variable immunodeficiency. J Immunol 2003; 170: 2765-2775.

12. Bronson $\mathrm{P}$, Chang $\mathrm{D}$, Bhangale $\mathrm{T}$, et al. Common variants at PVT1, ATG13-AMBRA1, AHI1 and CLEC16A are associated with selective IgA deficiency. Nat Amer 2016; 48: 1425-1429.

13. Karaca NE, Severcan EU, Guven B, et al. TNFRSF13B/TACI Alterations in Turkish Patients with Common Variable Immunodeficiency and IgA Deficiency. Avicenna J Med Biotechnol 2018; 10: 192-195.

14. Karaca NE, Severcan EU, Bilgin BG, et al. Familial inheritance and screening of first-degree relatives in common variable immunodeficiency and immunoglobulin A deficiency patients. Int J Immunopathol Pharmacol 2018; 32: 2058738418779458.

15. Woof JM, Kerr MA. Immunoglobulin A: Molecular Mechanisms of Function and Role in Immune Defence. J Pathol 2013; 208: 270-282.

16. Woof JM, Kerr MA. The function of immunoglobulin A in immunity. J Pathol 2006; 208: 270-282. 
17. Ganal-Vonarburg S, Macpherson AJ. IgA-about the unexpected. J Exp Med 2018; 215: 1965-1966.

18. Mantis J, Rol N, Corthésy B. Secretory IgA’s Complex Roles in Immunity and Mucosal Homeostasis in the Gut. Mucosal Immunol 2011; 4: 603-611.

19. Bakema JE, van Egmond M. The human immunoglobulin A Fc receptor FcaRI: a multifaceted regulator of mucosal immunity. Mucosal Immunol 2011; 4: 612-624.

20. Hansen IS, Baeten D, Dunnen J. The inflammatory function of human IgA. Cell Mol Life Sci 2019; 76: 1041-1055.

21. Berrón-Ruiz L, López-Herrera G, Vargas-Hernández A, et al. Lymphocytes and B-cell abnormalities in patients with common variable immunodeficiency (CVID). Allergol Immunopathol 2014; 42: 35-43.

22. Litzman J, Vlková M, Pikulová Z, et al. T and B lymphocyte subpopulations and activation/differentiation markers in patients with selective IgA deficiency. Clin Exp Immunol 2006; 147: 249-254.

23. Yel L. Selective IgA deficiency. J Clin Immunol 2010; 30: 10-16.

24. Warnatz K, Denz A, Drager R, et al. Severe deficiency of switched memory B cells $(\mathrm{CD} 27(+) \operatorname{IgM}(-) \operatorname{IgD}(-))$ in subgroups of patients with common variable immunodeficiency: a new approach to classify a heterogeneous disease. Blood 2002; 99: 1544-1551.

25. Nechvatalova J, Pavlik T, Litzman J, et al. Terminally differentiated memory $\mathrm{T}$ cells are increased in patients with common variable immunodeficiency and selective IgA deficiency. Centr Eur J Immunol 2017; 42: 244-251.

26. Castigli E, Wilson SA, Garibyan L, et al. TACI is mutant in common variable immunodeficiency and IgA deficiency. Nat Genet 2005; 37: 829-834.

27. Lemarquis AL, Einarsdottir HK, Kristjansdottir RN, et al. Transitional B Cells and TLR9 Responses Are Defective in Selective IgA Deficiency. Front Immunol 2018; 9: 909.

28. Asano T, Kaneko H, Terada T, et al. Molecular analysis of B-cell differentiation in selective or partial IgA deficiency. Clin Exp Immunol 2004; 136: 284-290.

29. Yazdani R, Fatholahi M, Ganjalikhani-Hakemi M, et al. Role of apoptosis in common variable immunodeficiency and selective immunoglobulin A deficiency. Mol Immunol 2016; 71: 1-9.

30. Bateman EA, Ayers L, Sadler R, et al. T cell phenotypes in patients with common variable immunodeficiency disorders: associations with clinical phenotypes in comparison with other groups with recurrent infections. Clin Exp Immunol 2012; 170: 202-211.

31. Rothblum-Oviatt C, Wright J, Lefton-Greif MA, et al. Ataxia telangiectasia: a review. Orphanet J Rare Dis 2016; 11: 159.

32. Derfalvi B, Maurer K, McDonald McGinn DM, et al. B cell development in chromosome 22q11.2 deletion syndrome. Clin Immunol 2016; 163: 1-9.

33. Macpherson AJ, Geuking MB, McCoy KD. Immunoglobulin A: a bridge between innate and adaptive immunity. Curr Opin Gastroenterol 2011; 27: 529-533.

34. Gutzeit C, Magri G, Cerutti A. Intestinal IgA production and its role in host-microbiome interaction. Immunol Rev 2014; 260: 76-85.

35. Macpherson AJ, Köller Y, McCoy KD. The bilateral responsiveness between intestinal microbes and IgA. Trends Immunol 2015; 36: 460-470.

36. Donaldson GP, Ladinsky MS, Yu KB, et al. Gut microbiota utilize immunoglobulin A for mucosal colonization. Science 2018; 360 : 795-800.

37. Fadlallah J, El Kafsi H, Sterlin D, et al. Microbial ecology perturbation in human IgA deficiency. Sci Transl Med 2018; 10: pii: eaan1217.

38. Palm NW, de Zoete MR, Cullen TW, et al. Immunoglobulin A coating identifies colitogenic bacteria In inflammatory bowel disease. Cell 2014; 158: 1000-1010.

39. Bienvenu F, Anghel SI, Duvanel CB, et al. Early diagnosis of celiac disease in IgA deficient children: contribution of a point-of-care test. BMC Gastroenterol 2014; 14: 186.
40. Pallav $\mathrm{K}, \mathrm{Xu} \mathrm{H}$, Leffler DA, et al. Immunoglobulin A deficiency in Celiac Disease in the United States. J Gastroenterol Hepatol 2016; 31: 133-137.

41. Cinetto F, Scarpa R, Rattazzi M, et al. The broad spectrum of lung diseases in primary antibody deficiencies. Eur Respir Rev 2018; 27 : pii: 180019.

42. Jesenak M, Banovcin P, Jesenakova B, et al. Pulmonary manifestations of primary immunodeficiency disorders in children. Front Pediatr 2014; $2: 77$.

43. Pasternak G, Lewandowicz-Uszyńska A, Pentoś K. Disorders of Humoral Immunity in Children with IgG Subclass Deficiency and Recurrent Respiratory Infections. Adv Exp Med Biol 2018; 1108: 99-106.

44. Aghamohammadi A, Cheraghi T, Gharagozlou M, et al. IgA deficiency: correlation between clinical and immunological phenotypes. J Clin Immunol 2009; 29: 130-136.

45. De Oliveira-Serra FA, Mosca T, Santos de Menezes MD, et al. Clinical symptoms in IgA deficiency. Rev Alerg Mex 2017; 64: 34-39.

46. Hodkinson J, Bangs C, Wartenberg-Demand A, et al. Low IgA and IgM Is Associated with a Higher Prevalence of Bronchiectasis in Primary Antibody Deficiency. J Clin Immunol 2017; 37: 329.

47. Schussler E, Beasley MB, Maglione PJ. Lung disease in primary antibody deficiencies. J Allergy Clin Immunol Pract 2016; 4: 1039-1042.

48. Erkoçoğlu M, Civelek E, Kocabaş CN. Unusual presentation: Concurrent IgA deficiency and idiopathic pulmonary hemosiderosis. Pediatr Pulmonol 2016; 51: E34-E36.

49. Özcan C, Metin A, Erkoçoğlu M, et al. Bronchial hyperreactivity in children with antibody deficiencies. Allergol Immunopathol (Madr) 2015; 43: 57-61.

50. Özcan C, Metin A, Erkoçoğlu M, et al. Allergic diseases in children with primary immunodeficiencies. Turk J Pediatr 2014; 56: 41-47.

51. Urm SH, Yun HD, Fenta YA, et al. Asthma and risk of selective IgA deficiency or common variable immunodeficiency: a population-based case-control study. Mayo Clin Proc 2013; 88: 813-821.

52. Szczawińska-Popłonyk A, Komasińska P, Bręborowicz A. IgA deficiency: a risk factor for food allergy-related atopic dermatitis in infants and young children. Adv Dermatol Allergol 2016; XXXIII: 369-374.

53. van de Ven AAJM, Janssen WJM, Schulz LS, et al. Increased Prevalence of Gastrointestinal Viruses and Diminished Secretory Immunoglobulin a Levels in Antibody Deficiencies. J Clin Immunol 2014; 34: 962-970.

54. Aarason GJ, Jorgensen GH, Ludviksson BR. Primary immunodeficiency and autoimmunity: lessons from human diseases. Scand J Immunol 2010; 71: 317-328.

55. Jacob CM, Pastorino AC, Fahl K, et al. Autoimmunity in IgA deficiency: revisiting the role of $\operatorname{IgA}$ as a silent housekeeper. J Clin Immunol 2008; 28: 56-61.

56. Sparchez M, Lupan I, Delean D, et al. Primary complement and antibody deficiencies in autoimmune rheumatologic diseases with juvenile onset: a prospective study at two centers. Pediatr Rheumatol 2015; 13: 51.

57. Carneiro-Sampaio M, Coutinho A. Early-onset autoimmune disease as a manifestation of primary immunodeficiency. Front Immunol 2015; 6: 185.

58. Asada Y, Isomoto H, Shikuwa S, et al. Development of ulcerative colitis during the course of rheumatoid arthritis: Association with selective IgA deficiency. World J Gastroenterol 2006; 12: 5240-5243.

59. Mirpuri J, Raetz M, Sturge CR, et al. Proteobacteria-specific IgA regulates maturation of the intestinal microbiota. Gut Microbes 2014; 5: 28-39.

60. Soheili H, Abolhassani H, Arandi N, et al. Evaluation of natural regulatory $\mathrm{T}$ cells in subjests with selective IgA deficiency: from senior idea to novel opportunities. Int Arch Allergy Immunol 2013; 160: 208-214. 